\title{
A Single-shot Field Measurement Procedure for Radiated Emissions using Equivalent Surface Dipoles
}

This paper was downloaded from TechRxiv (https://www.techrxiv.org).

\section{LICENSE}

CC BY 4.0

SUBMISSION DATE / POSTED DATE

05-08-2021 / 09-08-2021

\section{CITATION}

binda, pankaj; Mitharwal, Rajendra; Singh, Ghanshyam (2021): A Single-shot Field Measurement Procedure for Radiated Emissions using Equivalent Surface Dipoles. TechRxiv. Preprint.

https://doi.org/10.36227/techrxiv.15111906.v1

$\mathrm{DOI}$

10.36227/techrxiv.15111906.v1 


\title{
A Single-shot Field Measurement Procedure for Radiated Emissions using Equivalent Surface Dipoles
}

\author{
Pankaj Binda, Student Member, IEEE, Rajendra Mitharwal, Member, IEEE, \\ and Ghanshyam Singh, Senior Member, IEEE
}

\begin{abstract}
The new generation of communication devices operating in higher frequency bands is constantly pushing the complexity of field measurements for electromagnetic compliance and the design of field probes. These compliance are to be met for both electric and magnetic fields which demands repeating the same measurement procedure using different probes. The main objective of this paper is to provide a procedure which provides both fields in a single shot measurement using a single probe based on source reconstruction algorithm. The algorithm is based on a novel way of placing single dipole per point tangentially to a fictitious surface based on surface equivalence theorem instead of three orthogonal dipoles per point in earlier works. The control of overall accuracy by varying dipole and measurement point density is also demonstrated. We also prove the existence, uniqueness and the error bounds involved in the inverse problem rigorously. The numerical results corroborates the effectiveness of the proposed procedure in obtaining accurate fields and also locating the regions of the Device Under Test responsible for overshooting the interference limits.
\end{abstract}

Index Terms-EMI, EMC, Field Measurments, Radiated Emissions, DUT, Surface Equivalence Theorem.

\section{INTRODUCTION}

$\mathbf{T}$ HE ever increasing demand of bandwidth of new generation communication devices poses a new challenge to the well established electromagnetic interference and electromagnetic compatibility (EMI-EMC) measurements and testing [1]. These EMC measurement standards are regularly updated for higher operating frequency range [2], [3]. The military standard MIL-STD $461 \mathrm{G}$ prescribe strict limits for the radiated electromagnetic fields compared to the commercial ones. The example of two Radiated Emissions tests under this standard are RE101 and RE102 for the radiated magnetic and electric fields by a Device Under Test (DUT). The electric and magnetic fields are measured using electric and magnetic probes connected to a spectrum analyzer or a vector network analyzer. The electric and magnetic probes are realized using dipole, loop, biconical or horn antennas. In order to measure both electric and magnetic fields radiated by a device, the EMC engineer may have to repeat the same experiment twice using different probes. This is still fine if the focus is on one single frequency. This is not the case in real scenarios where a range

Pankaj Binda, Rajendra Mitharwal and Ghanshyam Singh are with the Department of ECE, Malaviya National Institute of Technology, Jaipur, Rajasthan, 302017 INDIA e-mail: 2019rec9143@mnit.ac.in, rajendra.ece@mnit.ac.in and gsingh.ece@mnit.ac.in.

Manuscript received July 31, 2021; revised September XX, 20XX. of frequencies should be swept in order for the device to be a truly compliant with the standards.

As the high speed and miniaturization of digital circuit becomes the need of the hour, the requirement of high precision near field measurement systems [4] becomes more prominent. The measurement of near field is a key step in not only deciding the compliance of a DUT but also in characterizing the properties of antennas [5]. It requires very huge investments in terms of anechoic chambers [6]. However, such high cost technique is not an absolute necessity for EMIEMC measurements as the objective here is not to find the accurate radiation field patterns (as in the case of antennas) instead it is to find the maximum value of the field at a spherical measurement surface enclosing the DUT accurately. The near field measurements are obtained using electric and magnetic probes. The size and the design of probes specifically dictate the sensitivity and the spatial resolution [7], [8] for obtaining accurate field measurements. Apart from this, the EMC practitioner also need good calibration techniques [9][11]. These calibration techniques are further classified into spatial and frequency domain methods [10].

The design and fabrication of an electric probe are relatively easier compared to a magnetic probe. However, as the frequency goes higher the miniaturization of the electric probe deteriorates its sensitivity. The magnetic probe requires complex designs as frequency increases. The sensitivity is also an issue which can still be solved using the resonant probes [12]. However, the resonant effects limit their application to narrowband near field measurements. They need high pass and notch filters for explicitly suppressing the interference from the electric fields [13]. The magnetic probe with very high sensitivity, wideband operating frequency range and ability to suppress unwanted electric fields can still be realized. However, it increases the fabrication cost tremendously [14]. There is a trade off between the sensitivity and fabrication cost when it comes to choosing an appropriate probe.

These limitations can be addressed by performing near field measurements of the DUT and obtaining the field computationally at the location specified by the EMI-EMC standard. The near field measurement based source reconstruction algorithms broadly can be grouped under dipoles based, integral equations, Finite Element Method (FEM) and Finite Difference Method (FDM). The integral equations and dipole based method give greater accuracy as compared to the other methods [15], [16]. There also have been recent develop- 
ments where these methods are combined with optimization techniques [17] and regularization for broadband frequency range [18]. The dipole based method have limitations in terms of position and density of the dipoles to be fixed a priori [16]. However, the method is flexible enough to use new measurement devices [19]. The source reconstruction based on FDM [20] require simultaneous near field measurements on three different planes and a Perfectly Electrical Conductor (PEC) enclosure to correctly predict the radiated emissions. Moreover, the usage of FDM inherits its shortcomings in terms of numerical errors [21]. The source reconstruction algorithm in [22] based on the Finite Element Method (FEM) has also been used to predict the far field in the context of EMI and identifying the location of interference from multiple source [23] based on the estimation of s-parameters. The success of these methods is based on the phase measurement where recently promising low cost alternatives are proposed [24], [25].

In this paper, we address some of the limitations highlighted above with the proposed single-shot field measurement based on the equivalent surface dipoles based source reconstruction algorithm. The proposed method places only one dipole per point compared to three dipoles placed by the other dipole based methods [26]. The advantage of the dipoles based algorithm compared to other techniques lies in its faster implementation. The novel contribution of this work are that both electric and magnetic field are obtained using a single measurement using either an electric or magnetic probe. Secondly, the dependence of accuracy of the measured field on the number of dipoles and measurement points is demonstrated. We rigorously prove the existence, uniqueness, and error bounds in the measured field using proposed procedure as the third contribution. Finally, we also demonstrate the effectiveness of the proposed method in locating the potential source of EMI in the DUT.

The paper is outlined as: the theoretical background and notation related to the electromagnetic fields of the dipole are set in the section II. The section III presents the proposed procedure with the mathematical analysis in terms of existence, uniqueness and error bounds. The results validating the proposed procedure are presented in section IV followed by the conclusion in section $\mathrm{V}$.

\section{BACKGROUND AND NOTATION}

An infinitesimally small electric dipole, in a high-frequency regime, can be considered as a localized oscillating source. Specifically, we can represent the associated charge and current density as a function of space and time as $\rho(\boldsymbol{r}, t)=$ $\rho(\boldsymbol{r}) e^{-i \omega t}$ and $\boldsymbol{J}(\boldsymbol{r}, t)=\boldsymbol{J}(\boldsymbol{r}) e^{-i \omega t}$. The real physical quantities are obtained by taking the real part of these complex functions.

These functions induce their time variation on the electromagnetic potentials and fields. The vector potential $\boldsymbol{A}(\boldsymbol{r}, t)=$ $\boldsymbol{A}(\boldsymbol{r}) e^{-i \omega t}$ [27] where

$$
\boldsymbol{A}(\boldsymbol{r})=\frac{\mu_{0}}{4 \pi} \int_{\Omega} \boldsymbol{J}\left(\boldsymbol{r}^{\prime}\right) G\left(\boldsymbol{r}, \boldsymbol{r}^{\prime}\right) d \Omega^{\prime},
$$

with $\mu_{0}$ being the free space magnetic permeability, $\Omega$ being the support of the volume current density, $G\left(\boldsymbol{r}, \boldsymbol{r}^{\prime}\right)=\frac{e^{i k\left|\boldsymbol{r}-\boldsymbol{r}^{\prime}\right|}}{\left|\boldsymbol{r}-\boldsymbol{r}^{\prime}\right|}$ being the high frequency green's function with the wave number $k$. The magnetic and electric field intensity are linked to the vector potential by $\boldsymbol{H}=\frac{1}{\mu_{0}} \nabla \times \boldsymbol{A}$ and $\boldsymbol{E}=\frac{i \eta}{k} \nabla \times \boldsymbol{H}$ respectively. The wave impedance of free space is represented by $\eta$. The equation (1) can be further simplified by using the volume charge density $\rho$, the current continuity equation $\nabla \cdot \boldsymbol{J}=i \omega \rho$ to

$$
\boldsymbol{A}(\boldsymbol{r})=\frac{\mu_{0}}{4 \pi} \frac{e^{i k r}}{r} \int_{\Omega} \boldsymbol{J}\left(\boldsymbol{r}^{\prime}\right) d \Omega^{\prime} .
$$

The separation of the terms $\boldsymbol{r}$ and $\boldsymbol{r}^{\prime}$ into $\left|\boldsymbol{r}-\boldsymbol{r}^{\prime}\right|=r-\hat{r} \cdot \boldsymbol{r}^{\prime}$ is based on the assumption of infinitesimally small size of dipole. Using the tensorial identity $\partial_{j}\left(\boldsymbol{J}_{j} \boldsymbol{r}_{i}\right)=\left(\partial_{3} \boldsymbol{J}_{j}\right) \boldsymbol{r}_{i}+\boldsymbol{J}_{i}=$ $i \omega \rho+\boldsymbol{J}_{i}$, we get $\int_{\Omega} \boldsymbol{J}\left(\boldsymbol{r}^{\prime}\right) d \Omega^{\prime}=-\int \boldsymbol{r}^{\prime}\left(\nabla^{\prime} \cdot \boldsymbol{J}\left(\boldsymbol{r}^{\prime}\right)\right) d \Omega^{\prime}=$ $-i \omega \int \boldsymbol{r}^{\prime} \rho\left(\boldsymbol{r}^{\prime}\right) d \Omega^{\prime}$. The final expression of the vector potential relevant for this work is

$$
\boldsymbol{A}(\boldsymbol{r})=-\frac{i \omega \mu_{0}}{4 \pi} \boldsymbol{p} \frac{e^{i k r}}{r}
$$

where $\boldsymbol{p}=\int_{\Omega} \boldsymbol{r}^{\prime} \rho\left(\boldsymbol{r}^{\prime}\right) d \Omega^{\prime}$ is the electric dipole moment. This notation captures the alignment of the dipole in the vector $\boldsymbol{p}$. Moreover, simplified models can be obtained for $\boldsymbol{p}$ being a constant or linearly varying vector quantity. The magnetic and electric field intensity are obtained using standard vector calculus identities [27] as

$$
\begin{aligned}
\boldsymbol{H}(\boldsymbol{r}) & =\frac{c k^{2}}{4 \pi}(\hat{\boldsymbol{r}} \times \boldsymbol{p}) \frac{e^{i k r}}{r}\left(1-\frac{1}{i k r}\right) \\
\boldsymbol{E}(\boldsymbol{r}) & =\frac{1}{4 \pi \epsilon_{0}} k^{2}(\hat{\boldsymbol{r}} \times \boldsymbol{p}) \times \hat{\boldsymbol{r}} \frac{e^{i k r}}{r} \\
& +[3 \hat{\boldsymbol{r}}(\hat{\boldsymbol{r}} \cdot \boldsymbol{p})-\boldsymbol{p}]\left(\frac{1}{r^{3}}-\frac{i k}{r^{2}}\right) e^{i k r}
\end{aligned}
$$

The above expressions are used to define a set of dipoles on fictitious surface based on surface equivalence theorem. Consider a source $S$ which radiates the electromagnetic field $(\boldsymbol{E}(\boldsymbol{r}), \boldsymbol{H}(\boldsymbol{r}))$ in the three-dimensional space $\mathbb{R}^{3}$. The space $\mathbb{R}^{3}$ is divided into two domains such that $S \in \Omega$ and $\bar{\Omega} \cup \Omega_{e} \in \mathbb{R}^{3}$. If we replace the original field in $\Omega$ as $\left(\boldsymbol{E}_{i}(\boldsymbol{r}), \boldsymbol{H}_{i}(\boldsymbol{r})\right)$ then the surface equivalence surface allows us to replace the original source $S$ with equivalent surface currents $(\boldsymbol{J}(\boldsymbol{r}), \boldsymbol{M}(\boldsymbol{r}))$ on the boundary surface $\Gamma_{s}=\partial \Omega$ which radiate $(\boldsymbol{E}(\boldsymbol{r}), \boldsymbol{H}(\boldsymbol{r}))$ in the $\Omega_{e}$ and the electromagnetic fields different from $(\boldsymbol{E}(\boldsymbol{r}), \boldsymbol{H}(\boldsymbol{r})$ ) (usually set to zero according to Love's condition) in the region $\Omega$. The objective of reconstructing $(\boldsymbol{E}(\boldsymbol{r}), \boldsymbol{H}(\boldsymbol{r}))$ can be achieved using a single current density function (either $\boldsymbol{J}(\boldsymbol{r})$ or $\boldsymbol{M}(\boldsymbol{r})$ ). These current densities satisfy

$$
\begin{aligned}
\hat{n} \times\left[\boldsymbol{H}(\boldsymbol{r})-\boldsymbol{H}_{i}(\boldsymbol{r})\right] & =\boldsymbol{J}(\boldsymbol{r}) \\
-\hat{n} \times\left[\boldsymbol{E}(\boldsymbol{r})-\boldsymbol{E}_{i}(\boldsymbol{r})\right] & =\boldsymbol{M}(\boldsymbol{r})
\end{aligned}
$$

for $r \in \Gamma_{s}$. 


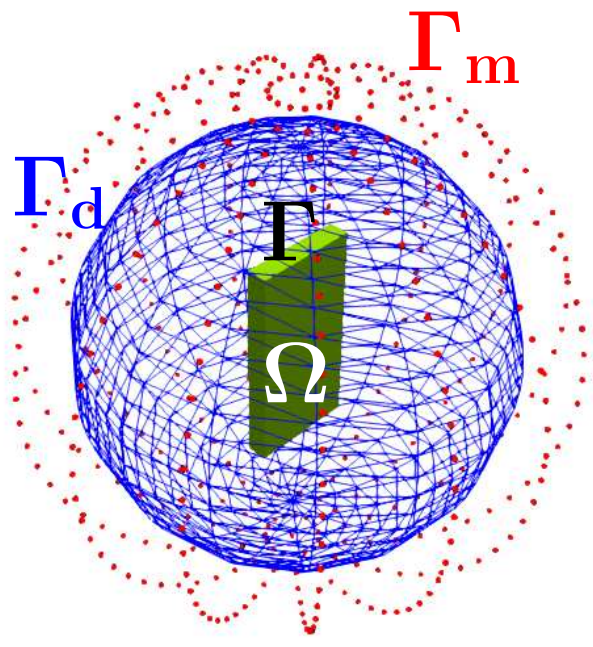

Fig. 1: Measurement setup.

\section{Proposed Single-Shot Field Measurement PROCEDURE}

Consider a typical measurement setup for estimating the high frequency radiation emissions from a DUT as shown in Figure 1. The DUT is represented by the volume region $\Omega$. The boundary of the DUT is defined by $\Gamma=\partial \Omega$. The area of interest where the electromagnetic fields are assessed or computed is the region exterior to $\Omega$. The region is represented by $\Omega_{e}=\mathbb{R}^{3} / \bar{\Omega}$ where $\bar{\Omega}=\Omega \cup \Gamma$ is a closed region.

The reconstruction of radiated electromagnetic fields by the DUT requires the flow of either electric or magnetic currents or both on an equivalent surface. These currents are proposed to be represented by a set of electric dipoles placed on the equivalent surface $\Gamma_{d}$. The magnetic field and electric field due to each electric dipole in this set can be easily computed using the equation (4) and (5). Each dipole is placed tangentially to the surface $\Gamma_{d}$ which ensures the applicability of the surface equivalence principle.

The density of electric dipoles is dictated by the minimum number of samples required to discretize the equivalent surface currents. The criteria is based on the number of minimum modes required in the spherical harmonics based expansions of a given electromagnetic field [5]. The number of samples per unit wavelength $k \lambda$ is roughly taken to be 10 per unit wavelength [28]. The convex hull of the DUT is chosen as the equivalent surface $\Gamma_{d}$ (a spherical surface for point sources such as dipoles). The equivalent surface $\Gamma_{d}$ is discretized into a triangular mesh with the average edge length specified as one-tenth of the wavelength corresponding to the operating frequency. On each edge, we define an electric dipole placed at the midpoint and align its moment $\boldsymbol{p}$ along the edge. The set of electric dipoles represents the equivalent surface dipoles decomposing the electromagnetic field for $r \in \Omega_{e}$ as

$$
\begin{aligned}
& \boldsymbol{E}(\boldsymbol{r})=\sum_{j=1}^{N_{d}} p_{j} \boldsymbol{E}_{j}(\boldsymbol{r}) \\
& \boldsymbol{H}(\boldsymbol{r})=\sum_{j=1}^{N_{d}} p_{j} \boldsymbol{H}_{j}(\boldsymbol{r})
\end{aligned}
$$

where $N_{d}$ is the total number of dipoles, $\boldsymbol{E}_{j}(\boldsymbol{r})=E_{j x}(\boldsymbol{r}) \hat{x}+$ $E_{j y}(\boldsymbol{r}) \hat{y}+E_{j z}(\boldsymbol{r}) \hat{z}$ and $\boldsymbol{H}_{j}(\boldsymbol{r})=H_{j x}(\boldsymbol{r}) \hat{x}+H_{j y}(\boldsymbol{r}) \hat{y}+$ $H_{j z}(\boldsymbol{r}) \hat{z}$ are the electric and magnetic field of the $j^{t h}$ dipole respectively computed using equation (4) and (5). The next step is to define a set of measurement points $\left\{\boldsymbol{r}_{i}\right\}_{i=1}^{N_{m}}$ lying on the measurement surface $\Gamma_{m}$. This naturally transforms equations (7) and (8) into the rectangular system of linear equations as

$$
\begin{aligned}
\mathbf{T p} & =\mathbf{e}, \\
\mathbf{K p} & =\mathbf{h},
\end{aligned}
$$

where element wise $\mathbf{p}_{j}=p_{j}$ are the unknown coefficients, the element of the electric field system matrix is defined as

$$
\mathbf{T}_{i j}= \begin{cases}E_{j x}\left(\boldsymbol{r}_{i}\right), & \text { if } 1 \leq i \leq N_{m} \\ E_{j y}\left(\boldsymbol{r}_{i}\right), & \text { if } N_{m}+1 \leq i \leq 2 N_{m} \\ E_{j z}\left(\boldsymbol{r}_{i}\right), & \text { if } 2 N_{m}+1 \leq i \leq 3 N_{m}\end{cases}
$$

and the magnetic field system matrix

$$
\mathbf{K}_{i j}=\left\{\begin{array}{ll}
H_{j x}\left(\boldsymbol{r}_{i}\right), & \text { if } 1 \leq i \leq N_{m} \\
H_{j y}\left(\boldsymbol{r}_{i}\right), & \text { if } N_{m}+1 \leq i \leq 2 N_{m} \\
H_{j z}\left(\boldsymbol{r}_{i}\right), & \text { if } 2 N_{m}+1 \leq i \leq 3 N_{m}
\end{array} .\right.
$$

The right hand side vectors element wise are given by

$$
\mathbf{e}_{i}=\left\{\begin{array}{ll}
E_{x}\left(\boldsymbol{r}_{i}\right), & \text { if } 1 \leq i \leq N_{m} \\
E_{y}\left(\boldsymbol{r}_{i}\right), & \text { if } N_{m}+1 \leq i \leq 2 N_{m} \\
E_{z}\left(\boldsymbol{r}_{i}\right), & \text { if } 2 N_{m}+1 \leq i \leq 3 N_{m}
\end{array},\right.
$$

and

$$
\mathbf{h}_{i}=\left\{\begin{array}{ll}
H_{x}\left(\boldsymbol{r}_{i}\right), & \text { if } 1 \leq i \leq N_{m} \\
H_{y}\left(\boldsymbol{r}_{i}\right), & \text { if } N_{m}+1 \leq i \leq 2 N_{m} \\
H_{z}\left(\boldsymbol{r}_{i}\right), & \text { if } 2 N_{m}+1 \leq i \leq 3 N_{m}
\end{array},\right.
$$

where $\boldsymbol{E}\left(\boldsymbol{r}_{i}\right)=E_{x}\left(\boldsymbol{r}_{i}\right) \hat{x}+E_{y}\left(\boldsymbol{r}_{i}\right) \hat{y}+E_{z}\left(\boldsymbol{r}_{i}\right) \hat{z}$ and $\boldsymbol{H}\left(\boldsymbol{r}_{i}\right)=$ $H_{x}\left(\boldsymbol{r}_{i}\right) \hat{x}+H_{y}\left(\boldsymbol{r}_{i}\right) \hat{y}+H_{z}\left(\boldsymbol{r}_{i}\right) \hat{z}$ are the measured electromagnetic fields of the DUT at the measurement point $\boldsymbol{r}_{i}$.

\section{A. Procedure for Single-shot Field Measurements}

For a DUT, whose electromagnetic field data is of interest can be obtained as follows:

i) Measure the electric field $\boldsymbol{E}(\boldsymbol{r})$ at a predefined set of measurement points $\left\{\boldsymbol{r}_{i}\right\}_{i=1}^{N_{m}}$ and set $\boldsymbol{e}$ vector according to equation (13). If the magnetic field measurements are chosen as an initial step then set the vector according to the equation (14).

ii) Obtain the unknown coefficients i.e the dipole moments for the set of dipoles using the equation (9) (or equation (10) if magnetic field is being measured in the initial step) using the inverse method outlined in the following subsection.

iii) Compute the magnetic field at the measurement points set $\left\{\boldsymbol{r}_{i}\right\}_{i=1}^{N_{m}}$ using equation (10) (or equation (9) if magnetic field measurements are chosen initially). 


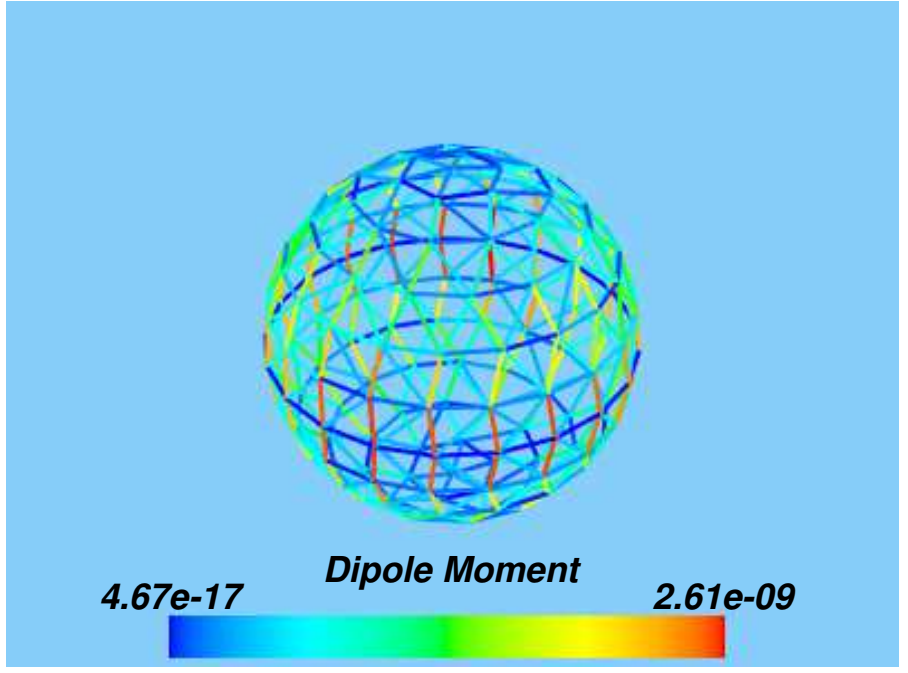

Fig. 2: Dipole Antenna: Reconstructed dipole moment of the antenna aligned along $\mathrm{z}$ axis.

\section{B. Existence \& Uniqueness of the Solution}

The existence of the surface current density $\boldsymbol{J}$ replacing the existing DUT is proved using the surface equivalence principle. Assume that the DUT is replaced with an equivalent surface $\Gamma_{d}$ that encloses a volume region $\Omega_{d}$. The solution provides a surface current density $\boldsymbol{J}(\boldsymbol{r})$ on $\Gamma_{d}$ such that it radiates the original electromagnetic field $(\boldsymbol{E}(\boldsymbol{r}), \boldsymbol{H}(\boldsymbol{r}))$ in the region $\mathbb{R}^{3} / \Omega_{d}$. Let us assume that such a $\boldsymbol{J}(\boldsymbol{r})$ does not exist. The absence of $\boldsymbol{J}(\boldsymbol{r})$ implies the existence of the original electromagnetic field across the surface $\Gamma_{d}$. This contradicts the assumption that the DUT is replaced with an equivalent surface. Hence, there exists a $\boldsymbol{J}(\boldsymbol{r})$ on $\Gamma_{d}$ that will radiate $(\boldsymbol{E}(\boldsymbol{r}), \boldsymbol{H}(\boldsymbol{r}))$ in the region $\mathbb{R}^{3} / \Omega_{d}$.

The uniqueness of the solution current density is ensured due to the Surface Equivalence Principle. Consider the DUT replaced with the equivalent surface $\Gamma_{d}$. The $\Gamma_{d}$ splits $\mathbb{R}^{3}$ into two regions $\mathbb{R}^{3} / \Omega_{d}$ and $\Omega_{d}$. Let us assume that there are two surface current densities $\boldsymbol{J}_{1}(\boldsymbol{r})$ and $\boldsymbol{J}_{2}(\boldsymbol{r})$ which radiate $(\boldsymbol{E}(\boldsymbol{r}), \boldsymbol{H}(\boldsymbol{r}))$ in $\mathbb{R}^{3} / \Omega_{d}$ and $\left(\boldsymbol{E}_{d}(\boldsymbol{r}), \boldsymbol{H}_{d}(\boldsymbol{r})\right)$ in the region $\Omega_{d}$. If a new current density $\boldsymbol{J}(\boldsymbol{r})=\boldsymbol{J}_{1}(\boldsymbol{r})-\boldsymbol{J}_{2}(\boldsymbol{r})$ is placed on $\Gamma_{d}$ then it radiates zero electromagnetic fields by principle of superposition. This is possible only if $\boldsymbol{J}(\boldsymbol{r})=0$ which implies $\boldsymbol{J}_{1}(\boldsymbol{r})=\boldsymbol{J}_{2}(\boldsymbol{r})$. Hence, the uniqueness of $\boldsymbol{J}(\boldsymbol{r})$ is ensured if $\boldsymbol{E}_{d}(\boldsymbol{r}), \boldsymbol{H}_{d}(\boldsymbol{r})$ is specified in the $\Omega_{d}$.

\section{Error Bounds}

The proposed procedure takes the input as the measured electric or magnetic field data and computes the second one at the predefined measurement points. In the analysis presented here we prove that the error of the computed field is upper bounded by the error of the measurement system.

Let the measured electric field $\boldsymbol{e}+\delta \boldsymbol{e}$ has an uncertainty $\delta \boldsymbol{e}$ causing deviation in the solution $p$. Due to $T$ being a linear operator, we get

$$
\mathbf{T}(\mathbf{p}+\delta \mathbf{p})=\mathbf{e}+\delta \mathbf{e}
$$

In the next step, the magnetic field is obtained as

$$
\mathbf{h}+\delta \mathbf{h}=\mathbf{K}(\mathbf{p}+\delta \mathbf{p})
$$

corrupted with the error. Subtracting (9) from (15) and equation (10) from (16) gives

$$
\begin{aligned}
\mathbf{T} \delta \mathbf{p} & =\delta \mathbf{e} \\
\delta \mathbf{h} & =\mathbf{K} \delta \mathbf{p} .
\end{aligned}
$$

Combining the above two equations, we get

$$
\delta \mathbf{h}=\mathbf{K} \mathbf{T}^{-1} \delta \mathbf{e} .
$$

Using the Cauchy-Schwarz inequality [29] we get

$$
\|\delta \mathbf{h}\|_{2} \leq\|\mathbf{K}\|_{2}\left\|\mathbf{T}^{-1}\right\|_{2}\|\delta \mathbf{e}\|_{2}
$$

when the electric field was chosen in the initial step. If magnetic field is measured in first step the inequality is

$$
\|\delta \mathbf{e}\|_{2} \leq\|\mathbf{T}\|_{2}\left\|\mathbf{K}^{-1}\right\|_{2}\|\delta \mathbf{h}\|_{2} .
$$

This clearly demonstrates that the computed field is bounded by the error in the input field measurement data. The interested reader may refer [30] and the references therein for more rigorous proofs based on Sobolev spaces.

\section{Solution of the Inverse Source Reconstruction}

The DUT replaced with equivalent surface dipoles results in the discretization of the inverse problem of source reconstruction. We demonstrate the method here using the electric field measurements taken as initial step. The arguments remain same for the magnetic field measurements. The linear system of equations is given by

$$
\mathrm{Tp}=\mathbf{e}
$$

The inverse problem finds the solution vector $\mathbf{p}$ by minimizing $\|\mathbf{T p}-\mathbf{e}\|_{2} /\|\mathbf{e}\|_{2}$. The number of unknowns $N_{d}$ is the number of dipoles placed on the equivalent surface $\Gamma_{d}$. The matrix $\mathbf{T}$ has the dimension of $3 N_{m}$ rows and $N_{d}$ columns. The choice of $N_{d}$ is dependent on the discretization criteria of $\lambda / 10$ edge length.

Since the measurement surface $\Gamma_{m}$ encloses $\Gamma_{d}$, usually the discretization criteria will result in the $N_{m}>N_{d}$. The strict adherence to the criteria will result in the oversampling of the field at $\Gamma_{m}$. The existence of non-radiating and minimum energy modes when the field is modeled at $\Gamma_{d}$ causes these fields to be evanescent at the $\Gamma_{m}$ [31]. This is the cause of the ill-posedness when the equation (22) is solved. These evanescent modes are responsible for the existence of the null space of $\mathbf{T}$. The equation (22) should be solved in the least square sense with Tikhonov's regularization [32]. The other techniques such as Truncated SVD and pseudoinverse can also be used. These techniques, however, become computationally prohibitive whenever the size of the problem increases. In such scenarios, we need to resort to iterative solvers which are also well suited for fast algorithms [32]. 


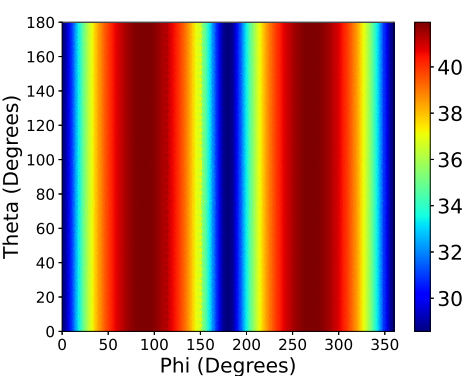

(a) $\boldsymbol{E}(\boldsymbol{r})$ : Original field data

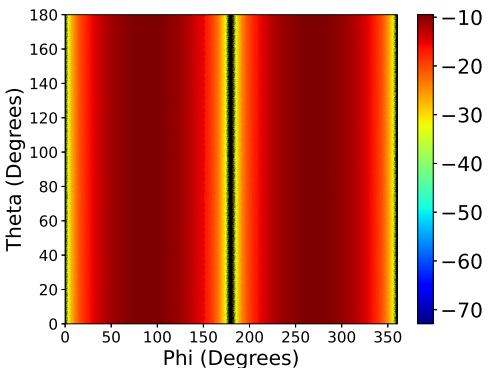

(d) $\boldsymbol{H}(\boldsymbol{r})$ : Original field data

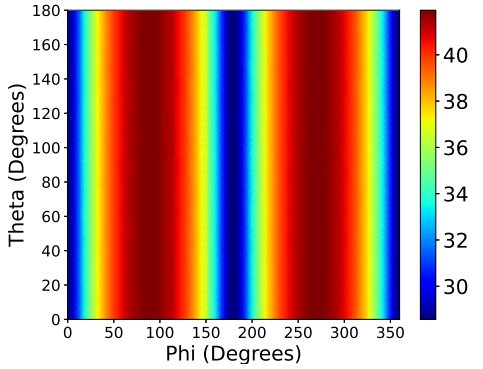

(b) $\boldsymbol{E}(\boldsymbol{r})$ : This work

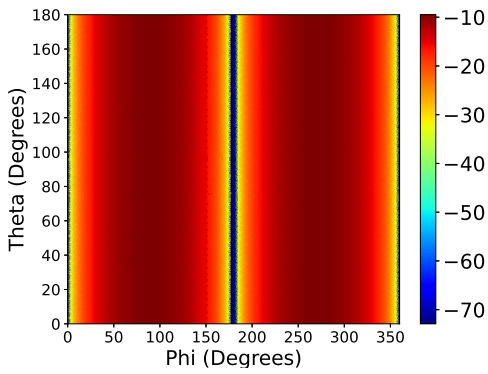

(e) $\boldsymbol{H}(\boldsymbol{r})$ : This work

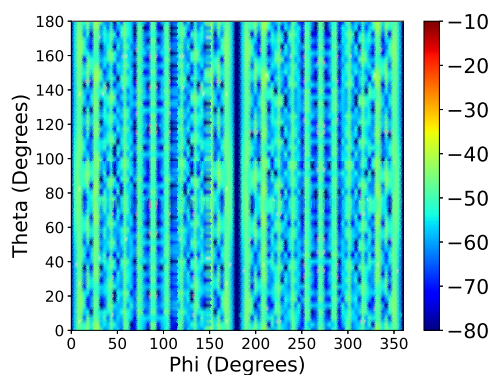

(c) $\boldsymbol{E}(\boldsymbol{r})$ : Relative error

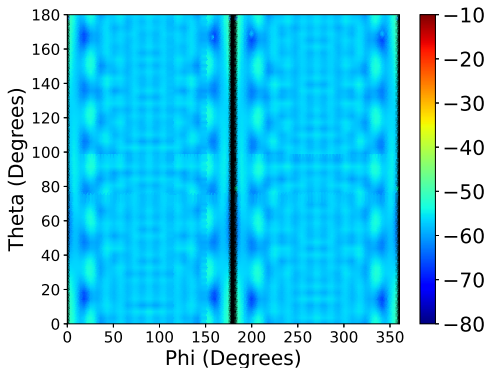

(f) $\boldsymbol{H}(\boldsymbol{r})$ : Relative error

Fig. 3: Dipole Antenna: Electric field $(\mathrm{dBV} / \mathrm{m})$, magnetic field $(\mathrm{dBA} / \mathrm{m})$ and relative error $(\mathrm{dB})$ in the field using proposed technique at $0.3 \mathrm{GHz}$ for the antenna aligned along $\mathrm{z}$ axis.

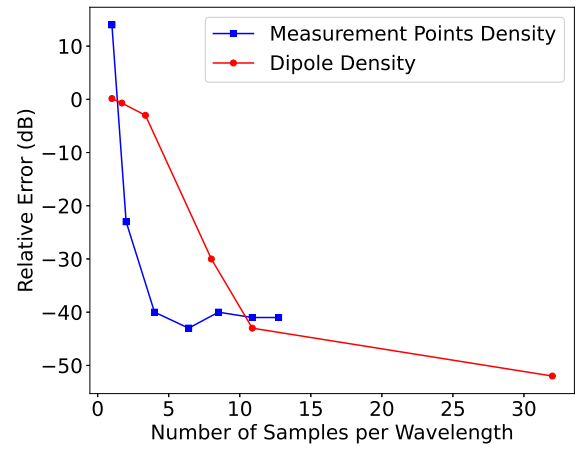

Fig. 4: Dipole Antenna: Variation of relative error in reconstructed field w.r.t. dipole density and measurement points at $0.3 \mathrm{GHz}$ for an antenna aligned along $\mathrm{z}$ axis.

To use iterative solvers, we need to convert the linear system of equation (22) to a normal residual system of equations

$$
\mathbf{T}^{\mathrm{H}} \mathbf{T p}=\mathbf{T}^{\mathrm{H}} \mathbf{e}
$$

where $\mathbf{T}^{\mathbf{H}}$ is the Hermitian transpose of $\mathbf{T}$. In the case of an underdetermined system, the normal error system of the equations are employed. The choice of the iterative solver, does not effect the solution accuracy significantly for the proposed algorithm from implementation points of view, the standard libraries for solving the least square problem can be used [33].

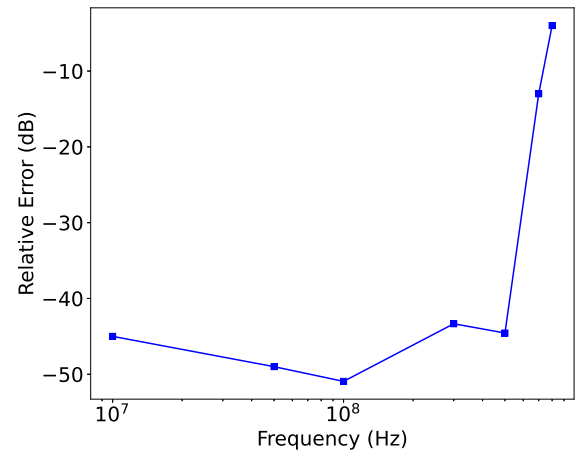

Fig. 5: Dipole Antenna: Variation of relative error in reconstructed field w.r.t. frequency for an antenna aligned along $\mathrm{z}$ axis.

\section{RESULTS AND VALIDATION}

The single-shot field measurement procedure is first being validated by an infinitesimally small dipole antenna. This source is chosen for validation because exact mathematical expressions for the radiated electromagnetic field are available. The role of dipole density on the equivalent surface $\Gamma_{d}$, the density of measurement points on the measurement surface $\Gamma_{m}$ and the variation of frequency on the overall accuracy of field is analyzed. The section concludes with the results of a realistic example of an horn antenna as the DUT. The accuracy of the proposed procedure is analyzed in terms of relative error in the measured field. The relative error at a 


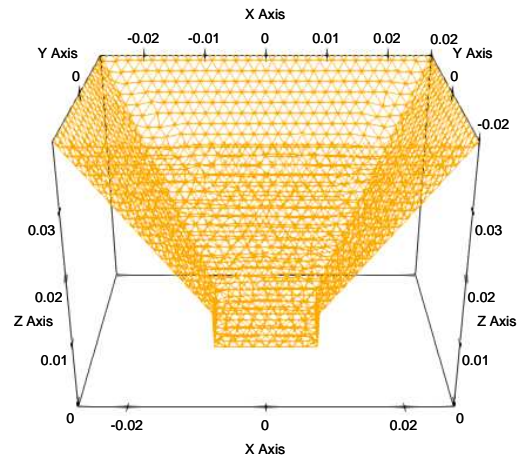

Fig. 6: Horn Antenna: Geometry with dimensions in metres.

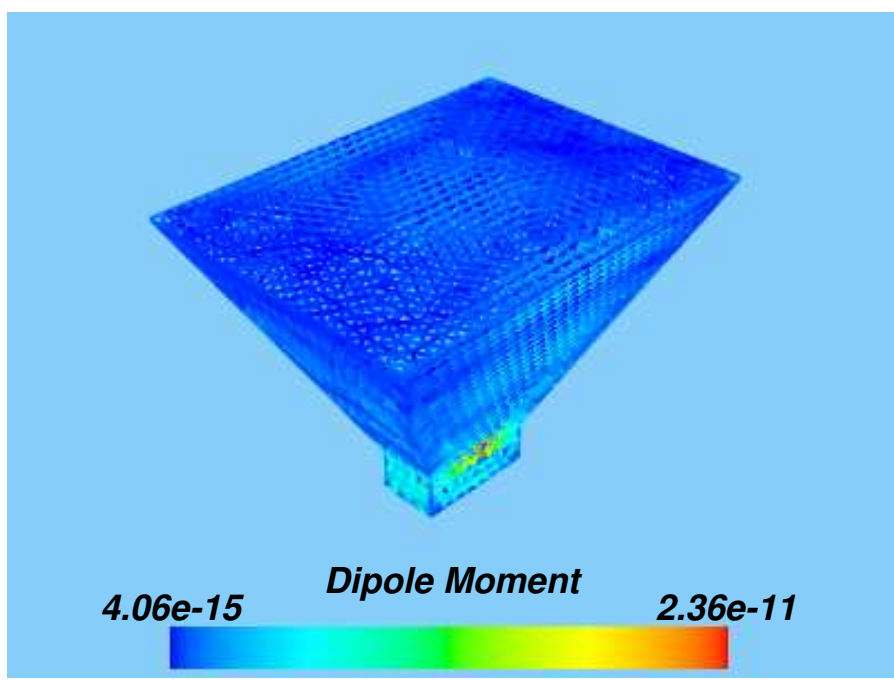

Fig. 7: Horn Antenna: Reconstructed dipole moment of the antenna aligned along $\mathrm{z}$ axis.

measurement point $\boldsymbol{r}_{i}$ is defined as

$$
\delta \boldsymbol{E}\left(\boldsymbol{r}_{i}\right)=\frac{\left|\boldsymbol{E}\left(\boldsymbol{r}_{i}\right)-\boldsymbol{E}_{m}\left(\boldsymbol{r}_{i}\right)\right|}{\left\|\boldsymbol{E}\left(\boldsymbol{r}_{i}\right)\right\|_{2}} .
$$

The effectiveness of the iterative solver in finding the solution of the inverse source reconstruction problem is determined based on the residual defined as

$$
\delta r=\frac{\|\mathbf{A} \mathbf{x}-\mathbf{b}\|}{\|\mathbf{b}\|}
$$

where $\mathbf{A}$ can be $\mathbf{T}$ or $\mathbf{K}$ based on the experiment.

\section{A. Validation: Dipole Antenna as DUT}

A dipole antenna is placed at the origin aligned along $\mathrm{z}$ axis operating at $0.3 \mathrm{GHz}$. We use the expressions for the fields given in [34] to ensure implementation differences with the equations (4) and (5). The dipole source is replaced with the equivalent surface $\Gamma_{d}$ represented by a spherical mesh of radius $1 \mathrm{~m}$ as shown in Figure 2. The equivalent surface dipoles are placed on each edge of the mesh (total edges being 495) corresponding to $\Gamma_{d}$. The measurement surface $\Gamma_{m}$, a spherical surface of radius $1.5 \mathrm{~m}$, is discretized with 5000 spherically equispaced points with 50 and 100 points along the $\theta$ and $\phi$ coordinates respectively. The discretization of both $\Gamma_{d}$ and $\Gamma_{m}$ corresponds to 10 samples per wavelength. This results in over-determined linear system of equations which are solved using Least Square Minimum Residual (LSMR) method [35] with the tolerance set at $10^{-6}$.

In the first case, the magnetic field data is used to find the unknown dipole moments on $\Gamma_{d}$ using 3064 LSMR iterations. The iterative solver finds the unknown dipole moments for the 495 surface dipole with the residual $\delta r=4.45 \times 10^{-3}$ using the $\mathbf{K}$ as system matrix. The electric field is then obtained using the matrix $\mathbf{T}$. The obtained electric field is compared in Figure $3 \mathrm{a}$ to $3 \mathrm{c}$. The electric field computed from the proposed technique matches perfectly with the original electric field. The maximum relative error in the set of measurement points is -41 $\mathrm{dB}$. This maximum value is observed for those measurement points which has minimum valued original electric field where as for the peak values the observed relative error is of order $-70 \mathrm{~dB}$.

Second case deals with the electric field data as the input column vector to obtain the unknown dipole moments. The solution is obtained in 2755 iterations with the residual $\delta r=$ $3.91 \times 10^{-3}$ using $T$ matrix. The magnetic field computed using the proposed technique is compared with the original magnetic field data in Figure 3d to $3 \mathrm{f}$. The maximum value of error $-50.92 \mathrm{~dB}$ is observed in the chosen set of measurement points. These results evidently demonstrate the validity of the proposed procedure in obtaining single-shot measurements of electromagnetic field data.

\section{B. Analysis: Role of dipole density, measurement point density in accuracy}

The question of the dipole density or the density of the measurement points is typically based on trial and error [36]. Using the proposed procedure, the obtained results confirm the criteria of $k d+N_{c}$ samples per wavelength [5] in deciding the dipole density where $d$ is the diameter of $\Omega$ and $N_{c}$ is a constant value.

The Figure 4 shows the variation of the relative error with respect to the number of samples needed per wavelength both for dipole density and measurement point density. For the curve corresponding to the dipole density, the measurement points are kept at 10 samples per wavelength and the number of dipoles are varied on the surface $\Gamma_{d}$. In other words, the average edge length of the mesh of the surface $\Gamma_{d}$ is varied. The curve clearly demonstrates that when the edge length of the mesh is $\lambda$, the relative error is quite high. Once the average edge length reaches $\lambda / 10$ the relative error improves. The further decrease in the average edge length saturates the error. Hence, for the best results with lower computational complexity it is better to keep the average edge length of the discretized $\Gamma_{d}$ as $\lambda / 10$. Next, the average edge length of discretized $\Gamma_{d}$ is kept constant at $\lambda / 10$ and the measurement point density of $\Gamma_{m}$ is varied to obtain the curve corresponding to measurement point density in Figure 4. Here also we see that the best values for the relative error is obtained when the number of points per wavelength is approximately 10 . 


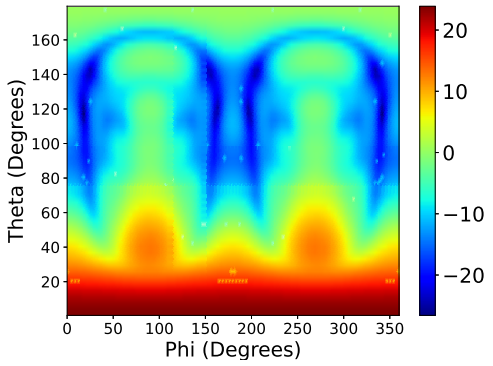

(a) $\boldsymbol{E}(\boldsymbol{r})$ : Original field data

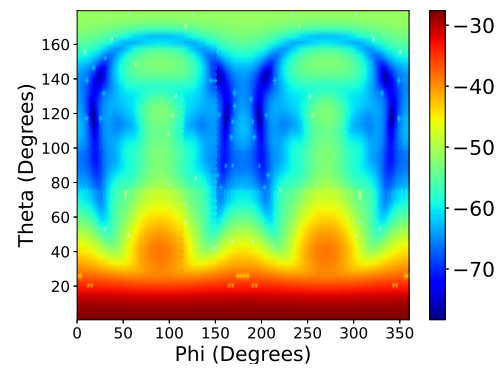

(d) $\boldsymbol{H}(\boldsymbol{r})$ : Original field data

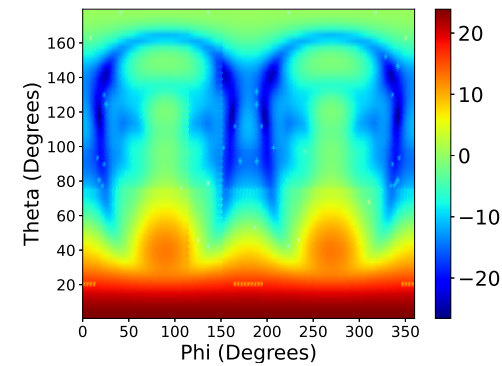

(b) $\boldsymbol{E}(\boldsymbol{r})$ : This work

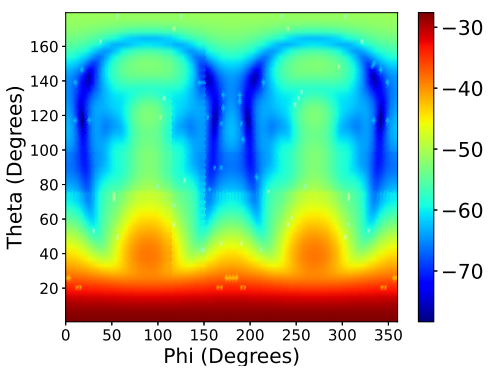

(e) $\boldsymbol{H}(\boldsymbol{r})$ : This work

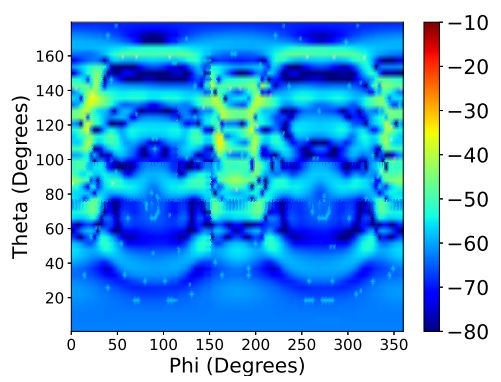

(c) $\boldsymbol{E}(\boldsymbol{r})$ : Relative error

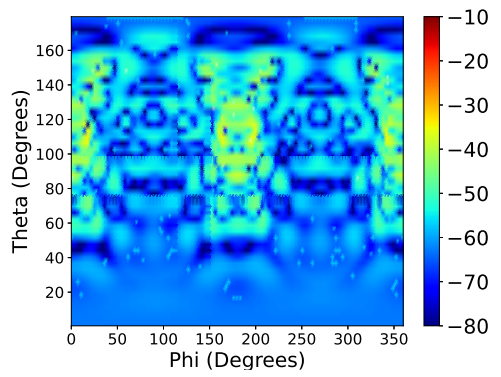

(f) $\boldsymbol{H}(\boldsymbol{r})$ : Relative error

Fig. 8: Horn Antenna: Electric field $(\mathrm{dBV} / \mathrm{m})$, magnetic field $(\mathrm{dBA} / \mathrm{m})$ and relative error $(\mathrm{dB})$ in the field using proposed technique at $14.125 \mathrm{GHz}$ for the antenna aligned along $\mathrm{z}$ axis.

After this, increasing the measurement point density keeps the relative error more or less around the same value. The role of the 10 samples per wavelength are further confirmed in the Figure 5 where the measurement setup is kept the same as in the previous subsection. The operating frequency is varied. As the plot clearly shows the error confined within a small range till the average edge length corresponds to $\lambda / 10$. The further increase deteriorates the accuracy of the obtained field values.

\section{Realistic scenario: Horn antenna as DUT}

In most realistic scenarios, the DUT's radiation pattern typically resembles that of isotropic radiators. Here, we choose the horn antenna because of its high directivity with the dimensions shown in Figure 6. This ensures in a wide dynamic range of the field values which brings numerical challenges for the inverse source reconstruction algorithms [32]. The operating frequency of the horn antenna is $14.125 \mathrm{GHz}$. The equivalent surface for this horn antenna is the convex hull $\Gamma_{d}$ shown in Figure 7. The number of dipoles placed on $\Gamma_{d}$ is 6402. The electric and magnetic field data of the horn antenna is generated synthetically using integral equations based solver [37] as shown in Figure 8a and 8d respectively. A voltage source of 1 Volts is applied at one of the edge at the input wave-guide as a voltage gap. The number of measurement points (placed equispaced along $\theta$ and $\phi$ ) on a spherical surface of radius $0.5 \mathrm{~m}$ is 5000 . The linear system of equations obtained in this scenario are solved using the LSMR iterative scheme with tolerance of $10^{-6}$.

The first case deals with the magnetic field data as an input to the proposed procedure. The problem took 2765 LSMR iterations with the residual $\delta r$ as $4 \times 10^{-3}$ using the system matrix $\mathbf{K}$. The electric field obtained in this case matches with the original field data as shown in Figure 8a to 8c. The maximum relative error is $-33 \mathrm{~dB}$ which exists in the minimal regions of the original electric field as can be seen in Figure $8 \mathrm{c}$. The maximal regions of electric field has a lower error of order of $-70 \mathrm{~dB}$.

In next experiment, the electric field data is used as an input to the proposed procedure. The system matrix $T$ is used to find the unknown dipole coefficients with 1388 LSMR iterations and residual $\delta r=3.94 \times 10^{-3}$. The maximum relative error in this case is $-34 \mathrm{~dB}$ as can be seen in Figure 8f. The obtained magnetic field in Figure 8e shows good match with the original magnetic field in Figure 8f.

The advantage of the proposed procedure is seen in Figure 7 where the high radiating regions of the DUT can be distinctly identified. This demonstrate that the proposed procedure potentially can serve as a tool for practicing EMC-EMI engineers for diagnostics purpose. This will help in addressing the electromagnetic compliance issues of the DUTs by identifying and rectifying the high radiating regions.

\section{CONClusion}

The paper presents a novel procedure for obtaining both electric and magnetic field in a single shot measurement. The procedure is based on the equivalent surface dipoles based source reconstruction algorithm. The source reconstruction is different from previously reported approaches as it places the dipoles tangentially on an equivalent surface. The theoretical analysis of error bounds on the obtained field is also presented. The numerical results validates the effectiveness of the proposed procedure in obtaining the fields for radiated 
emissions and identifying the radiating regions for resolving the electromagnetic compliance issues of a DUT.

\section{ACKNOWLEDGMENT}

Pankaj Binda would like to thank the Malaviya National Institute of Technology Jaipur for providing the institute's scholarship for his PhD work. The authors are grateful to Prof. Bajrang Bali for all his support.

\section{REFERENCES}

[1] K. Wiklundh and P. Stenumgaard, "EMC challenges for the era of massive internet of things," IEEE Electromagnetic Compatibility Magazine vol. 8, no. 2, pp. 65-74, 2019.

[2] MIL-STD-461G, "Requirements for the control of electromagnetic interference characteristics of subsystems and equipment," Department of Defense, U.S.A., 2015.

[3] A. C63.5-2017, "American national standard for electromagnetic compatibility-radiated emission measurements in electromagnetic interference (EMI) control-calibration and qualification of antennas (9 khz to $40 \mathrm{ghz}), "$ (Revision of ANSI C63.5-2005), pp. 1-114, 2017.

[4] J. Wang, Z. Yan, C. Fu, Z. Ma, and J. Liu, "Near-field precision measurement system of high-density integrated module," IEEE Transactions on Instrumentation and Measurement, vol. 70, pp. 1-9, 2021.

[5] J. E. Hansen, Spherical near-field antenna measurements. IET, 1988, vol. 26.

[6] R. De Leo and V. M. Primiani, "Radiated immunity tests: reverberation chamber versus anechoic chamber results," IEEE transactions on instrumentation and measurement, vol. 55, no. 4, pp. 1169-1174, 2006.

[7] M. Kanda, "Standard probes for electromagnetic field measurements," IEEE Transactions on Antennas and Propagation, vol. 41, no. 10, pp. 1349-1364, 1993.

[8] F. Costa, E. Laboure, F. Forest, and C. Gautier, "Wide bandwidth, large ac current probe for power electronics and EMI measurements," IEEE Transactions on Industrial Electronics, vol. 44, no. 4, pp. 502-511, 1997.

[9] M. Judd, "Transient calibration of electric field sensors," IEE Proceedings-Science, Measurement and Technology, vol. 146, no. 3, pp. 113-116, 1999.

[10] J. Shi, M. A. Cracraft, K. P. Slattery, M. Yamaguchi, and R. E. DuBroff, "Calibration and compensation of near-field scan measurements," IEEE Transactions on Electromagnetic Compatibility, vol. 47, no. 3, pp. 642650, 2005.

[11] J. Zhang, K. W. Kam, J. Min, V. V. Khilkevich, D. Pommerenke, and J. Fan, "An effective method of probe calibration in phase-resolved nearfield scanning for EMI application," IEEE transactions on instrumentation and measurement, vol. 62, no. 3, pp. 648-658, 2012

[12] J.-M. Kim, W.-T. Kim, and J.-G. Yook, "Resonance-suppressed magnetic field probe for EM field-mapping system," IEEE transactions on microwave theory and techniques, vol. 53, no. 9, pp. 2693-2699, 2005.

[13] Y.-T. Chou and H.-C. Lu, "Magnetic near-field probes with high-pass and notch filters for electric field suppression," IEEE transactions on microwave theory and techniques, vol. 61, no. 6, pp. 2460-2470, 2013.

[14] H.-H. Chuang, G.-H. Li, E. Song, H.-H. Park, H.-T. Jang, H.-B. Park, Y.J. Zhang, D. Pommerenke, T.-L. Wu, and J. Fan, "A magnetic-field resonant probe with enhanced sensitivity for RF interference applications," IEEE transactions on electromagnetic compatibility, vol. 55, no. 6, pp. 991-998, 2013.

[15] Y. A. Lopéz, F. L.-H. Andrés, M. R. Pino, and T. K. Sarkar, "An improved super-resolution source reconstruction method," IEEE Transactions on Instrumentation and Measurement, vol. 58, no. 11, pp. 38553866, 2009.

[16] Y. Álvarez, M. Rodríguez, F. Las-Heras, and M. M. Hernando, "On the use of the source reconstruction method for estimating radiated EMI in electronic circuits," IEEE Transactions on Instrumentation and Measurement, vol. 59, no. 12, pp. 3174-3183, 2010.

[17] Q. Huang, L. Li, X. Yan, B. Bae, H. Park, C. Hwang, and J. Fan, "Mom-based ground current reconstruction in RFI application," IEEE Transactions on Electromagnetic Compatibility, vol. 60, no. 4, pp. 11211128,2018

[18] N. Hendijani, S. D. Gedney, R. McConnell, M. J. Roberts, J. C. Young and R. J. Adams, "A broad-band huygens surface source model for nearfield to near-field transformations," IEEE Transactions on Electromagnetic Compatibility, vol. 60, no. 4, pp. 926-936, 2017.
[19] K. Kwak, J. Kim, and J. Kim, "Near-field validation of dipole-moment model extracted from GTEM cell measurements and application to a real application processor," IEEE Transactions on Electromagnetic Compatibility, vol. 60, no. 2, pp. 423-434, 2017.

[20] H. Weng, D. G. Beetner, and R. E. DuBroff, "Prediction of radiated emissions using near-field measurements," IEEE transactions on electromagnetic compatibility, vol. 53, no. 4, pp. 891-899, 2011.

[21] S. D. Gedney, "Introduction to the finite-difference time-domain (fdtd) method for electromagnetics," Synthesis Lectures on Computational Electromagnetics, vol. 6, no. 1, pp. 1-250, 2011.

[22] X. Gao, J. Fan, Y. Zhang, H. Kajbaf, and D. Pommerenke, "Far-field prediction using only magnetic near-field scanning for EMI test," IEEE transactions on electromagnetic compatibility, vol. 56, no. 6, pp. 1335 1343, 2014.

[23] J. Pan, X. Gao, and J. Fan, "Identifying interference from multiple noise sources using only magnetic near fields," IEEE Transactions on Electromagnetic Compatibility, vol. 63, no. 2, pp. 580-588, 2021.

[24] S. Marathe, Z. Chen, K. Ghosh, H. Kajbaf, S. Frei, M. Sørensen, D. Pommerenke, and J. Min, "Spectrum analyzer-based phase measurement for near-field EMI scanning," IEEE Transactions on Electromagnetic Compatibility, vol. 62, no. 3, pp. 848-858, 2019.

[25] T. Li, V. Khilkevich, and D. Pommerenke, "Phase-resolved near-field scan over random fields," IEEE Transactions on Electromagnetic Compatibility, vol. 58, no. 2, pp. 506-511, 2016.

[26] J. Nie, S. Yang, and D. Su, "Spherical equivalent dipole array theory and its applications to complex electromagnetic system," IEEE Access, vol. 7, pp. 111 756-111 766, 2019.

[27] J. D. Jackson, "Classical electrodynamics," 1999.

[28] W. C. Chew, E. Michielssen, J. Song, and J.-M. Jin, Fast and efficient algorithms in computational electromagnetics. Artech House, Inc., 2001.

[29] P. D. Lax, Linear Algebra and Its Applications. Wiley-Interscience, 2007.

[30] N. P. Valdivia and E. Williams, "The reconstruction of surface tangential components of the electromagnetic field from near-field measurements," Inverse problems, vol. 23, no. 2, p. 785, 2007.

[31] E. A. Marengo and R. W. Ziolkowski, "Nonradiating and minimum energy sources and their fields: Generalized source inversion theory and applications," IEEE Transactions on Antennas and Propagation, vol. 48, no. 10 , pp. $1553-1562,2000$

[32] J. Kornprobst, J. Knapp, R. A. Mauermayer, O. Neitz, A. Paulus, and T. F. Eibert, "Accuracy and conditioning of surface-source based nearfield to far-field transformations," IEEE Transactions on Antennas and Propagation, 2021

[33] P. Virtanen, R. Gommers, T. E. Oliphant, M. Haberland, T. Reddy, D. Cournapeau, E. Burovski, P. Peterson, W. Weckesser, J. Bright et al., "SciPy 1.0: fundamental algorithms for scientific computing in python," Nature methods, vol. 17, no. 3, pp. 261-272, 2020.

[34] C. A. Balanis, Antenna theory: analysis and design. John wiley \& sons, 2015.

[35] D. C.-L. Fong and M. Saunders, "LSMR: An iterative algorithm for sparse least-squares problems," SIAM Journal on Scientific Computing, vol. 33, no. 5, pp. 2950-2971, 2011.

[36] Z. Yu, J. A. Mix, S. Sajuyigbe, K. P. Slattery, and J. Fan, "An improved dipole-moment model based on near-field scanning for characterizing near-field coupling and far-field radiation from an IC," IEEE Transactions on electromagnetic compatibility, vol. 55, no. 1, pp. 97-108, 2012.

[37] S. Rao, D. Wilton, and A. Glisson, "Electromagnetic scattering by surfaces of arbitrary shape," IEEE Transactions on antennas and propagation, vol. 30, no. 3, pp. 409-418, 1982. 